\title{
The Two Relativistic Rydberg Formulas of Suto and Haug: Further Comments
}

\author{
Espen Gaarder Haug \\ Norwegian University of Life Sciences, Ås, Norway \\ Email: espenhaug@mac.com
}

How to cite this paper: Haug, E.G. (2020) The Two Relativistic Rydberg Formulas of Suto and Haug: Further Comments. Journal of Modern Physics, 11, 1938-1949. https://doi.org/10.4236/jmp.2020.1112122

Received: October 9, 2020

Accepted: December 7, 2020

Published: December 10, 2020

Copyright $\odot 2020$ by author(s) and Scientific Research Publishing Inc. This work is licensed under the Creative Commons Attribution International License (CC BY 4.0).

http://creativecommons.org/licenses/by/4.0/

\begin{abstract}
In a recent paper, we [1] discussed that Suto [2] has pointed out an interesting relativistic extension of Rydberg's formula. In that paper, we had slightly misunderstood Suto's approach, something we will comment on further here. The relativistic Suto formula is actually derived from a theory where the standard relativistic momentum relation is changed. The relativistic Rydberg formula we presented and mistakenly thought was the same as Suto's formula is, on the other hand, derived to be fully consistent with the standard relativistic energy-momentum relation. Here we will point out the differences between the formulas and correct some errors in our previous paper. The paper should give deeper and better intuition about the Rydberg formula and what it represents.
\end{abstract}

\section{Keywords}

Rydberg's Formula, Relativistic Extension, Compton Wavelength

\section{Introduction}

A considerable number of papers have been published on relativistic corrections of the Rydberg states, see for example [3] [4]. However, the main focus of these papers tends to be corrections based on relativistic quantum mechanics. Even if relativistic quantum mechanics is very powerful, this seems to give a limited intuition on why the Rydberg formula is non-relativistic, and how we can adjust the Rydberg formula to make it relativistic. Here we will look directly at the Rydberg formula and how it can be modified based on special relativistic effects without looking into relativistic quantum mechanics. The relativistic quantum mechanical approach may be considerably better in prediction power, but the main advantage with the approach here is that it gives energy and additional intuition about the non-relativistic versus relativistic Rydberg states. 
Since we use a series of variables and parameters, we will start by providing a list of symbols (Table 1), as a preface to our paper.

The well-known Rydberg [5] formula is given by

$$
\frac{1}{\lambda}=R_{\infty} Z^{2}\left(\frac{1}{n_{1}^{2}}-\frac{1}{n_{2}^{2}}\right)
$$

where $R_{\infty}$ is Rydberg's constant, which has a value of $10,973,731.568160(21)$ $\mathrm{m}^{-1}$ (NIST CODATA 2018 value). Even though the formula is very simple, it is hard to gain much intuition from it. The Rydberg constant can be rewritten as

$$
\begin{gathered}
R_{\infty}=\frac{m_{e} e^{4}}{8 \epsilon_{0}^{2} h^{3} c} \\
R_{\infty}=\frac{\frac{\hbar}{\bar{\lambda}_{e}} \frac{1}{c}\left(\sqrt{\left.\frac{\hbar}{c} \sqrt{\alpha} \sqrt{10^{7}}\right)^{4}}\right.}{8 \epsilon_{0}^{2} h^{3} c} \\
R_{\infty}=\frac{\frac{\hbar^{3}}{\bar{\lambda}_{e}} \frac{1}{c^{3}} \alpha^{2}\left(10^{7}\right)^{2}}{8\left(\frac{1}{4 \pi c^{2} 10^{-7}}\right)^{2} h^{3} c}
\end{gathered}
$$

Table 1. Symbol list.

\begin{tabular}{cl}
\hline Symbol & Represents \\
\hline$h$ & Planck constant \\
$c$ & reduced Planck constant \\
$V$ & velocity of the electron \\
$Z$ & atomic number \\
$R_{\infty}$ & Rydberg constant \\
$n_{1}$ & the principal quantum number of an energy level \\
$n_{2}$ & the principal quantum number of an energy level for the atomic electron transition \\
$\epsilon_{0}$ & vacuum permittivity \\
$\alpha$ & Fine structure constant \\
$e$ & elementary charge \\
$\lambda$ & Photon wavelength \\
$\lambda_{e}$ & Compton wavelength electron \\
$\bar{\lambda}$ & Reduced Compton wavelength electron \\
$m_{e}$ & rest mass of electron \\
$m_{p}$ & rest mass of proton \\
$p$ & momentum \\
$E$ & energy \\
$E_{k}$ & kinetic energy \\
\hline &
\end{tabular}




$$
\begin{gathered}
R_{\infty}=\frac{\frac{\hbar^{3}}{\bar{\lambda}_{e}} \frac{1}{c^{3}} \alpha^{2}}{8 \frac{1}{16 \pi^{2} c^{4}} h^{3} c} \\
R_{\infty}=\frac{1}{2} \frac{\hbar}{h} \frac{1}{\bar{\lambda}_{e}} \alpha^{2} \\
R_{\infty}=\frac{1}{2} \frac{\frac{h}{2 \pi}}{h} \frac{1}{\bar{\lambda}_{e}} \alpha^{2} \\
R_{\infty}=\frac{\alpha^{2}}{2 \lambda_{e}}
\end{gathered}
$$

And we can rewrite this as

$$
R_{\infty}=\frac{\alpha^{2}}{2 \frac{h}{m_{e} c}}=\frac{\alpha^{2} m_{e} c}{2 h}
$$

(In the former paper, we had incorrectly used the Compton wavelength rather than the reduced Compton wavelength in the beginning of this derivation and thus we incorrectly got $R_{\infty}=\frac{\alpha^{2}}{2 \frac{h}{m_{e} c}}=\frac{\alpha^{2} m_{e} c}{4 \pi \hbar}$. However, we made another error further down that canceled this error out and therefore we obtained the right result with respect to the Rydberg formula.)

This is standard knowledge, so we have shown nothing new so far. Let us now replace this in Rydberg's formula, which gives

$$
\begin{gathered}
\frac{1}{\lambda}=\frac{\alpha^{2} m_{e} c}{2 h} Z^{2}\left(\frac{1}{n_{1}^{2}}-\frac{1}{n_{2}^{2}}\right) \\
h \frac{c}{\bar{\lambda}}=Z^{2}\left(\frac{1}{2} m_{e} \frac{\alpha^{2} c^{2}}{n_{1}^{2}}-\frac{1}{2} m_{e} \frac{\alpha^{2} c^{2}}{n_{2}^{2}}\right)
\end{gathered}
$$

where $\frac{\alpha^{2} c^{2}}{n_{1}^{2}}$ can be seen as $v_{1}^{2}$ and $\frac{\alpha^{2} c^{2}}{n_{2}^{2}}$ as $v_{2}^{2}$. In other words, we can write this as

$$
E=h \frac{C}{\lambda}=\frac{Z^{2}}{2 \pi}\left(\frac{1}{2} m_{e} v_{1}^{2}-\frac{1}{2} m_{e} v_{2}^{2}\right)
$$

Since $\frac{1}{2} m v^{2}$ is the well-known approximation of the kinetic energy when $v \ll c$ (the first term of a Taylor series approximation), the Rydberg formula is clearly non-relativistic. Even though this is known, we have not seen any relativistic extension of the formula before Suto's paper [2]. However, before we discuss his formula, we will briefly show how we arrived at our relativistic version of the Rydberg formula. Since $\frac{1}{2} m v^{2}$ is the approximation for $v \ll c$, we simply replaced this approximation by the full relativistic kinetic energy 
$E_{k}=m c^{2} \gamma-m c^{2}$, where, as usual, $\gamma=\frac{1}{\sqrt{1-v^{2} / c^{2}}}$. This gives

$$
\begin{aligned}
E & =\left(\frac{m_{e} c^{2}}{\sqrt{1-v_{1}^{2} / c^{2}}}-m_{e} c^{2}-\frac{m_{e} c^{2}}{\sqrt{1-v_{2}^{2} / c^{2}}}-m_{e} c^{2}\right) \\
& =\left(\frac{m_{e} c^{2}}{\sqrt{1-v_{1}^{2} / c^{2}}}-\frac{m_{e} c^{2}}{\sqrt{1-v_{2}^{2} / c^{2}}}\right)
\end{aligned}
$$

where $v_{1}=Z \alpha c / n_{1}$ and $v_{2}=Z \alpha c / n_{2}$, and we also have that $m_{e}=\frac{h}{\lambda_{e}} \frac{1}{c}$, where $\lambda_{e}$ is the Compton [6] wavelength of the electron. The equation can then be rewritten as

$$
\begin{gathered}
h \frac{c}{\lambda}=\left(\frac{\frac{h}{\lambda_{e}} c}{\sqrt{1-\frac{Z^{2} \alpha^{2}}{n_{1}^{2}}}}-\frac{\frac{h}{\lambda_{e}} c}{\sqrt{1-\frac{Z^{2} \alpha^{2}}{n_{2}^{2}}}}\right) \\
\frac{1}{\lambda}=\left(\frac{1}{\lambda_{e} \sqrt{1-\frac{Z^{2} \alpha^{2}}{n_{1}^{2}}}}-\frac{1}{\lambda_{e} \sqrt{1-\frac{Z^{2} \alpha^{2}}{n_{2}^{2}}}}\right)
\end{gathered}
$$

We mistakenly thought that the formula we presented in the last paper was the same as Suto's relativistic Rydberg formula. However, Suto's [2] relativistic formula is (his equation 48)

$$
\frac{1}{\lambda}=\left(\frac{1}{\lambda_{e} \sqrt{1+\frac{\alpha^{2}}{n_{2}^{2}}}}-\frac{1}{\lambda_{e} \sqrt{1+\frac{\alpha^{2}}{n_{1}^{2}}}}\right)
$$

where $\lambda$ is the photon wavelength, and $\lambda_{e}$ is the Compton wavelength of the electron.

While our new relativistic formula should be consistent with the standard energy momentum relation $E^{2}=p^{2} c^{2}+m^{2} c^{4}$, where $p$ is the momentum, the Suto formula is not consistent with this, but it is consistent with the modified energy momentum relation that he presented in the same paper as $m_{e}^{2} c^{4}-p_{n}^{2} c^{2}=m_{n}^{2} c^{4}$. The Taylor expansion of our relativistic formula is

$$
\begin{aligned}
\frac{1}{\bar{\lambda}}= & \frac{1}{\bar{\lambda}_{e}}\left(\left(1-\frac{Z^{2} \alpha^{2}}{2 n_{1}^{2}}+\frac{3 Z^{4} \alpha^{4}}{8 n_{1}^{4}}+\frac{5 Z^{6} \alpha^{6}}{16 n_{1}^{6}}+\cdots\right)\right. \\
& \left.-\left(1-\frac{Z^{2} \alpha^{2}}{2 n_{2}^{2}}+\frac{3 Z^{4} \alpha^{4}}{8 n_{2}^{4}}+\frac{5 Z^{6} \alpha^{6}}{16 n_{2}^{6}}+\cdots\right)\right)
\end{aligned}
$$

And the Taylor series expansion of Suto's formula is 


$$
\frac{1}{\bar{\lambda}}=\frac{1}{\bar{\lambda}_{e}}\left(\left(1-\frac{\alpha^{2}}{2 n_{1}^{2}}-\frac{3 \alpha^{4}}{8 n_{1}^{4}}+\frac{5 \alpha^{6}}{16 n_{1}^{6}}+\cdots\right)-\left(1-\frac{\alpha^{2}}{2 n_{2}^{2}}-\frac{3 \alpha^{4}}{8 n_{2}^{4}}+\frac{5 \alpha^{6}}{16 n_{2}^{6}}+\cdots\right)\right)
$$

We incorrectly pointed out that Suto might have made a mistake and there was a sign error in his series expansion, but this is actually not the case. This was because we thought his formula was identical to the one that we had derived ${ }^{1}$.

When expanded to hold for any atom, the Suto formula, based on his energy momentum assumption, must likely be:

$$
\frac{1}{\lambda}=\left(\frac{1}{\lambda_{e} \sqrt{1+\frac{Z^{2} \alpha^{2}}{n_{2}^{2}}}}-\frac{1}{\lambda_{e} \sqrt{1+\frac{Z^{2} \alpha^{2}}{n_{1}^{2}}}}\right)
$$

while our relativistic extension of the Rydberg formula is

$$
\frac{1}{\lambda}=\left(\frac{1}{\lambda_{e} \sqrt{1-\frac{Z^{2} \alpha^{2}}{n_{1}^{2}}}}-\frac{1}{\lambda_{e} \sqrt{1-\frac{Z^{2} \alpha^{2}}{n_{2}^{2}}}}\right)
$$

In other words, we have recently gotten two relativistic Rydberg formulas; one consistent with the standard relativistic energy momentum relation (the Haug formula) and one consistent with what we can call a somewhat alternative theory of Suto.

In general, we would think the formula that is consistent with the standard relativistic energy momentum relation is more correct and consistent. However, it is not necessarily easy to test out which one is superior, as the hydrogen atom is known to be best described by the relativistic Dirac [7] wave equation.

\section{Length Contraction and Length Expansion of the Compton Wavelength}

In the Suto formula, the Compton wavlength of the electron looks like it is extended in length due to velocity of the electron, since in the denominator we have $\lambda_{e} \sqrt{1+\frac{Z^{2} \alpha^{2}}{n_{2}^{2}}}$ and $\lambda_{e} \sqrt{1+\frac{Z^{2} \alpha^{2}}{n_{1}^{2}}}$, while in our relativistic formula we have standard relativistic length contraction, $\lambda_{e} \sqrt{1-\frac{Z^{2} \alpha^{2}}{n_{2}^{2}}}$ and $\lambda_{e} \sqrt{1-\frac{Z^{2} \alpha^{2}}{n_{1}^{2}}}$ of the Compton wavelength of the electron, as observed from the laboratory frame that the electron is moving relative to. We would find it strange if the Compton wave should undergo length expansion because of motion and not length contraction as measured with Einstein-Poincaré synchronized clocks. Einstein-Poincaré synchronized clocks are simply indicating we assume the one-way speed of light is the same as the round trip speed of light when we synchronize the clocks over ${ }^{1}$ We apologize for missing this, but we are taking steps to correct that here. 
distance. $^{2}$

It seems that the methodology we have laid out basically only takes into account that the electron moves parallel to the laboratory frame. In other words, our model seems to be 2-dimensional. However, if in reality the reduced Compton wavelength represents a radius of a sphere rather than a length, then this length contraction will likely be correct for electrons moving in any direction as observed from the laboratory frame with Einstein-Poincaré synchronized clocks. There could naturally be a series of other corrections needed to fit observations, such as relativistic quantum mechanical effects. There should also be several interesting angles to investigate further: how close we can get to predicting observations with non-quantum mechanical models, for example.

\section{Table Calculations}

In Table 2, we look at the Lyman series. This is for a hydrogen atom where we hold $n_{1}=1$ and in this table let $n_{2}$ vary from 2 to 7 . We see that the Haug formula predicts a slightly shorter wavelength than the non-relativistic Rydberg formula, while the Suto formula predicts a slightly longer wavelength than the non-relativistic formula. Table 3 shows predictions from the three formulas for the Balmer series, where we have $n_{1}=2$ and let $n_{2}$ vary from 3 to 7 . The first column in Table 3 is from real observations. The real observations in this case have been done in air, so we have adjusted all the three formulas by the refraction index in air; this simply means we need to divide the formulas by the refraction index in air, which is about 1.00029. One can find observation studies done in a vacuum and in air; when comparing theoretical predictions against observations it is naturally important to know how the observations have been done the medium matters.

Table 2. The table shows the Lyman series calculated from the non-relativistic formula, the Haug relativistic formula, and the Suto relativistic formula. The difference-column shows the difference in percent between the relativistic formula predictions and the non-relativistic formula predictions for the Haug and Suto formulas. The Haug relativistic formula predicts a slightly shorter wavelength than the non-relativistic formula, and the Suto formula predicts a slightly longer wavelength. The Haug formula seems to be consistent with relativistic length contraction (also of waves).

\begin{tabular}{cccccc}
\hline$n_{2}$ & Non-Relativistic & Haug & Difference & Suto & Difference \\
\hline 2 & 121.568 & 121.562 & $-0.0050 \%$ & 121.575 & $0.0050 \%$ \\
3 & 102.573 & 102.569 & $-0.0044 \%$ & 102.578 & $0.0044 \%$ \\
4 & 97.255 & 97.251 & $-0.0042 \%$ & 97.259 & $0.0042 \%$ \\
5 & 94.975 & 94.971 & $-0.0042 \%$ & 94.979 & $0.0042 \%$ \\
6 & 93.781 & 93.778 & $-0.0041 \%$ & 93.785 & $0.0041 \%$ \\
7 & 93.076 & 93.072 & $-0.0041 \%$ & 93.080 & $0.0041 \%$ \\
\hline
\end{tabular}

${ }^{2}$ Whether or not such synchronization is fully valid is an ongoing discussion, see for example [8] [9], but that is outside the scope of this paper. 
Table 3. The table shows the Balmer series calculated from the non-relativistic formula, the Haug relativistic formula, and the Suto relativistic formula. The difference column is the difference in percent between the relativistic formula predictions and the non-relativistic formula predictions for the Haug and Suto formulas. The Haug relativistic formula predicts a slightly shorter wavelength than the non-relativistic formula, and the Suto formula predicts a slightly longer wavelength. The Haug formula seems to be consistent with relativistic length contraction (also of waves). The observations are from the Atomic Spectra NIST Standard Reference Database 78 Version 5.7, and are done in air, so we have made an adjustment based on the refraction index in all formulas based on air. If this adjustment is not done, our prediction is far off, as expected.

\begin{tabular}{|c|c|c|c|c|c|c|c|}
\hline$n_{2}$ & $\begin{array}{l}\text { Observed } \\
\text { in air }\end{array}$ & $\begin{array}{l}\text { Non-relativistic } \\
\text { in air }\end{array}$ & Difference & Haug in air & Difference & Suto in air & Difference \\
\hline 3 & 656.28 & 656.279 & $-0.0001 \%$ & 656.270 & $-0.0016 \%$ & 656.289 & $0.0013 \%$ \\
\hline 4 & 486.13 & 486.133 & $0.0006 \%$ & 486.127 & $-0.0007 \%$ & 486.139 & $0.0018 \%$ \\
\hline 5 & 434.05 & 434.047 & $-0.0007 \%$ & 434.042 & $-0.0018 \%$ & 434.052 & $0.0005 \%$ \\
\hline 6 & 410.17 & 410.175 & $0.0011 \%$ & 410.170 & $0.0000 \%$ & 410.179 & $0.0022 \%$ \\
\hline 7 & 397.005 & 397.008 & $0.0009 \%$ & 397.004 & $-0.0002 \%$ & 397.013 & $0.0020 \%$ \\
\hline
\end{tabular}

Since Table 2 and Table 3 are covering hydrogen atoms, we have also adjusted all of the formulas by multiplying by one divided by the adjusted mass $\frac{m_{P}}{m_{P}+m_{e}}$, where $m_{P}$ is the proton mass, and $m_{e}$ is the electron mass. First of all, observations for the hydrogen atom are likely not accurate enough to distinguish between the non-relativistic and relativistic formulas, but we leave that for future discussion. For the hydrogen atom, the Lyman series is where the differences between the three formulas are the greatest, so there is no reason to look at the Paschen, Brackett, Humphreys, or Pfund series in addition, as the differences between non-relativistic and relativistic predictions would be even smaller. To test out the formulas, one would likely need to look at much heavier hydrogen-like atoms, as the electrons move much faster, in general, and therefore relativistic effects would play a bigger role. Still, one likely needs a relativistic wave equation to include all necessary adjustments, another issue to consider in this framework.

In our original table, there was a typo in the spreadsheet that resulted in incorrect values from our relativistic Rydberg formula. Below, we present the corrected tables (Table 4 and Table 5); here we have not adjusted the formulas based on the reduced mass, so the difference in values will be the same in any of the formulas, even if we multiply each predicted wavelength with one divided by the reduced mass: $\frac{m_{P}}{m_{P}+m_{e}}$.

\section{Conclusion}

We have shown that the relativistic extensions of the Rydberg formula given by Suto and Haug are two different formulas. The Haug relativistic Rydberg formula 
Table 4. The table shows the Rydberg formula predictions and the relativistic predictions for the first 137 elements. As we can see, the difference increases between the two models. Here we are just looking at the case $n_{1}=1$ and $n_{2}=2$.

\begin{tabular}{|c|c|c|c|c|c|c|c|c|c|}
\hline $\begin{array}{c}\text { Atomic } \\
\#\end{array}$ & $\begin{array}{l}\text { Rydberg } \\
\text { formula }\end{array}$ & $\begin{array}{c}\text { Relativistic } \\
\text { formula }\end{array}$ & Diff. & $\begin{array}{c}\text { Diff. } \\
\%\end{array}$ & $\begin{array}{c}\text { Atomic } \\
\#\end{array}$ & $\begin{array}{l}\text { Rydberg } \\
\text { formula }\end{array}$ & $\begin{array}{l}\text { Relativistic } \\
\text { formula }\end{array}$ & Diff. & $\begin{array}{c}\text { Diff. } \\
\%\end{array}$ \\
\hline 1 & 121.5023 & 121.4962 & $(0.0061)$ & $-0.005 \%$ & 71 & 0.0241 & 0.0181 & $(0.0060)$ & $-32.9 \%$ \\
\hline 2 & 30.3756 & 30.3695 & $(0.0061)$ & $-0.020 \%$ & 72 & 0.0234 & 0.0175 & $(0.0060)$ & $-34.2 \%$ \\
\hline 3 & 13.5003 & 13.4942 & $(0.0061)$ & $-0.045 \%$ & 73 & 0.0228 & 0.0168 & $(0.0060)$ & $-35.4 \%$ \\
\hline 4 & 7.5939 & 7.5878 & $(0.0061)$ & $-0.080 \%$ & 74 & 0.0222 & 0.0162 & $(0.0060)$ & $-36.8 \%$ \\
\hline 5 & 4.8601 & 4.8540 & $(0.0061)$ & $-0.125 \%$ & 75 & 0.0216 & 0.0156 & $(0.0060)$ & $-38.1 \%$ \\
\hline 6 & 3.3751 & 3.3690 & $(0.0061)$ & $-0.180 \%$ & 76 & 0.0210 & 0.0151 & $(0.0060)$ & $-39.5 \%$ \\
\hline 7 & 2.4796 & 2.4736 & $(0.0061)$ & $-0.245 \%$ & 77 & 0.0205 & 0.0145 & $(0.0060)$ & $-41.0 \%$ \\
\hline 8 & 1.8985 & 1.8924 & $(0.0061)$ & $-0.320 \%$ & 78 & 0.0200 & 0.0140 & $(0.0060)$ & $-42.5 \%$ \\
\hline 9 & 1.5000 & 1.4940 & $(0.0061)$ & $-0.406 \%$ & 79 & 0.0195 & 0.0135 & $(0.0060)$ & $-44.0 \%$ \\
\hline 10 & 1.2150 & 1.2090 & $(0.0061)$ & $-0.502 \%$ & 80 & 0.0190 & 0.0130 & $(0.0059)$ & $-45.6 \%$ \\
\hline 11 & 1.0042 & 0.9981 & $(0.0061)$ & $-0.607 \%$ & 81 & 0.0185 & 0.0126 & $(0.0059)$ & $-47.3 \%$ \\
\hline 12 & 0.8438 & 0.8377 & $(0.0061)$ & $-0.724 \%$ & 82 & 0.0181 & 0.0121 & $(0.0059)$ & $-49.0 \%$ \\
\hline 13 & 0.7189 & 0.7129 & $(0.0061)$ & $-0.9 \%$ & 83 & 0.0176 & 0.0117 & $(0.0059)$ & $-50.8 \%$ \\
\hline 14 & 0.6199 & 0.6138 & $(0.0061)$ & $-1.0 \%$ & 84 & 0.0172 & 0.0113 & $(0.0059)$ & $-52.7 \%$ \\
\hline 15 & 0.5400 & 0.5339 & $(0.0061)$ & $-1.1 \%$ & 85 & 0.0168 & 0.0109 & $(0.0059)$ & $-54.6 \%$ \\
\hline 16 & 0.4746 & 0.4686 & $(0.0061)$ & $-1.3 \%$ & 86 & 0.0164 & 0.0105 & $(0.0059)$ & $-56.6 \%$ \\
\hline 17 & 0.4204 & 0.4144 & $(0.0061)$ & $-1.5 \%$ & 87 & 0.0161 & 0.0101 & $(0.0059)$ & $-58.6 \%$ \\
\hline 18 & 0.3750 & 0.3689 & $(0.0061)$ & $-1.6 \%$ & 88 & 0.0157 & 0.0098 & $(0.0059)$ & $-60.8 \%$ \\
\hline 19 & 0.3366 & 0.3305 & $(0.0061)$ & $-1.8 \%$ & 89 & 0.0153 & 0.0094 & $(0.0059)$ & $-63.0 \%$ \\
\hline 20 & 0.3038 & 0.2977 & $(0.0061)$ & $-2.0 \%$ & 90 & 0.0150 & 0.0091 & $(0.0059)$ & $-65.3 \%$ \\
\hline 21 & 0.2755 & 0.2695 & $(0.0061)$ & $-2.2 \%$ & 91 & 0.0147 & 0.0087 & $(0.0059)$ & $-67.7 \%$ \\
\hline 22 & 0.2510 & 0.2450 & $(0.0061)$ & $-2.5 \%$ & 92 & 0.0144 & 0.0084 & $(0.0059)$ & $-70.2 \%$ \\
\hline 23 & 0.2297 & 0.2236 & $(0.0061)$ & $-2.7 \%$ & 93 & 0.0140 & 0.0081 & $(0.0059)$ & $-72.8 \%$ \\
\hline 24 & 0.2109 & 0.2049 & $(0.0061)$ & $-3.0 \%$ & 94 & 0.0138 & 0.0078 & $(0.0059)$ & $-75.5 \%$ \\
\hline 25 & 0.1944 & 0.1884 & $(0.0061)$ & $-3.2 \%$ & 95 & 0.0135 & 0.0075 & $(0.0059)$ & $-78.4 \%$ \\
\hline 26 & 0.1797 & 0.1737 & $(0.0061)$ & $-3.5 \%$ & 96 & 0.0132 & 0.0073 & $(0.0059)$ & $-81.3 \%$ \\
\hline 27 & 0.1667 & 0.1606 & $(0.0061)$ & $-3.8 \%$ & 97 & 0.0129 & 0.0070 & $(0.0059)$ & $-84.4 \%$ \\
\hline 28 & 0.1550 & 0.1489 & $(0.0061)$ & $-4.1 \%$ & 98 & 0.0127 & 0.0067 & $(0.0059)$ & $-87.6 \%$ \\
\hline 29 & 0.1445 & 0.1384 & $(0.0060)$ & $-4.4 \%$ & 99 & 0.0124 & 0.0065 & $(0.0059)$ & $-91.0 \%$ \\
\hline 30 & 0.1350 & 0.1290 & $(0.0060)$ & $-4.7 \%$ & 100 & 0.0122 & 0.0062 & $(0.0059)$ & $-94.6 \%$ \\
\hline 31 & 0.1264 & 0.1204 & $(0.0060)$ & $-5.0 \%$ & 101 & 0.0119 & 0.0060 & $(0.0059)$ & $-98.3 \%$ \\
\hline 32 & 0.1187 & 0.1126 & $(0.0060)$ & $-5.4 \%$ & 102 & 0.0117 & 0.0058 & $(0.0059)$ & $-102.2 \%$ \\
\hline 33 & 0.1116 & 0.1055 & $(0.0060)$ & $-5.7 \%$ & 103 & 0.0115 & 0.0056 & $(0.0059)$ & $-106.3 \%$ \\
\hline 34 & 0.1051 & 0.0991 & $(0.0060)$ & $-6.1 \%$ & 104 & 0.0112 & 0.0053 & $(0.0059)$ & $-110.6 \%$ \\
\hline 35 & 0.0992 & 0.0931 & $(0.0060)$ & $-6.5 \%$ & 105 & 0.0110 & 0.0051 & $(0.0059)$ & $-115.1 \%$ \\
\hline 36 & 0.0938 & 0.0877 & $(0.0060)$ & $-6.9 \%$ & 106 & 0.0108 & 0.0049 & $(0.0059)$ & $-119.9 \%$ \\
\hline 37 & 0.0888 & 0.0827 & $(0.0060)$ & $-7.3 \%$ & 107 & 0.0106 & 0.0047 & $(0.0059)$ & $-125.0 \%$ \\
\hline
\end{tabular}




\section{Continued}

\begin{tabular}{|c|c|c|c|c|c|c|c|c|c|}
\hline 38 & 0.0841 & 0.0781 & $(0.0060)$ & $-7.7 \%$ & 108 & 0.0104 & 0.0045 & $(0.0059)$ & $-130.4 \%$ \\
\hline 39 & 0.0799 & 0.0738 & $(0.0060)$ & $-8.2 \%$ & 109 & 0.0102 & 0.0043 & $(0.0059)$ & $-136.1 \%$ \\
\hline 40 & 0.0759 & 0.0699 & $(0.0060)$ & $-8.6 \%$ & 110 & 0.0100 & 0.0041 & $(0.0059)$ & $-142.1 \%$ \\
\hline 41 & 0.0723 & 0.0662 & $(0.0060)$ & $-9.1 \%$ & 111 & 0.0099 & 0.0040 & $(0.0059)$ & $-148.6 \%$ \\
\hline 42 & 0.0689 & 0.0628 & $(0.0060)$ & $-9.6 \%$ & 112 & 0.0097 & 0.0038 & $(0.0059)$ & $-155.4 \%$ \\
\hline 43 & 0.0657 & 0.0597 & $(0.0060)$ & $-10.1 \%$ & 113 & 0.0095 & 0.0036 & $(0.0059)$ & $-162.8 \%$ \\
\hline 44 & 0.0628 & 0.0567 & $(0.0060)$ & $-10.6 \%$ & 114 & 0.0093 & 0.0035 & $(0.0059)$ & $-170.7 \%$ \\
\hline 45 & 0.0600 & 0.0540 & $(0.0060)$ & $-11.2 \%$ & 115 & 0.0092 & 0.0033 & $(0.0059)$ & $-179.1 \%$ \\
\hline 46 & 0.0574 & 0.0514 & $(0.0060)$ & $-11.7 \%$ & 116 & 0.0090 & 0.0031 & $(0.0059)$ & $-188.3 \%$ \\
\hline 47 & 0.0550 & 0.0490 & $(0.0060)$ & $-12.3 \%$ & 117 & 0.0089 & 0.0030 & $(0.0059)$ & $-198.1 \%$ \\
\hline 48 & 0.0527 & 0.0467 & $(0.0060)$ & $-12.9 \%$ & 118 & 0.0087 & 0.0028 & $(0.0059)$ & $-208.9 \%$ \\
\hline 49 & 0.0506 & 0.0446 & $(0.0060)$ & $-13.5 \%$ & 119 & 0.0086 & 0.0027 & $(0.0059)$ & $-220.6 \%$ \\
\hline 50 & 0.0486 & 0.0426 & $(0.0060)$ & $-14.1 \%$ & 120 & 0.0084 & 0.0025 & $(0.0059)$ & $-233.4 \%$ \\
\hline 51 & 0.0467 & 0.0407 & $(0.0060)$ & $-14.8 \%$ & 121 & 0.0083 & 0.0024 & $(0.0059)$ & $-247.4 \%$ \\
\hline 52 & 0.0449 & 0.0389 & $(0.0060)$ & $-15.4 \%$ & 122 & 0.0082 & 0.0022 & $(0.0059)$ & $-263.0 \%$ \\
\hline 53 & 0.0433 & 0.0372 & $(0.0060)$ & $-16.1 \%$ & 123 & 0.0080 & 0.0021 & $(0.0059)$ & $-280.4 \%$ \\
\hline 54 & 0.0417 & 0.0357 & $(0.0060)$ & $-16.9 \%$ & 124 & 0.0079 & 0.0020 & $(0.0059)$ & $-299.9 \%$ \\
\hline 55 & 0.0402 & 0.0342 & $(0.0060)$ & $-17.6 \%$ & 125 & 0.0078 & 0.0018 & $(0.0059)$ & $-321.9 \%$ \\
\hline 56 & 0.0387 & 0.0327 & $(0.0060)$ & $-18.3 \%$ & 126 & 0.0077 & 0.0017 & $(0.0059)$ & $-347.1 \%$ \\
\hline 57 & 0.0374 & 0.0314 & $(0.0060)$ & $-19.1 \%$ & 127 & 0.0075 & 0.0016 & $(0.0060)$ & $-376.2 \%$ \\
\hline 58 & 0.0361 & 0.0301 & $(0.0060)$ & $-19.9 \%$ & 128 & 0.0074 & 0.0015 & $(0.0060)$ & $-410.2 \%$ \\
\hline 59 & 0.0349 & 0.0289 & $(0.0060)$ & $-20.8 \%$ & 129 & 0.0073 & 0.0013 & $(0.0060)$ & $-450.8 \%$ \\
\hline 60 & 0.0338 & 0.0278 & $(0.0060)$ & $-21.6 \%$ & 130 & 0.0072 & 0.0012 & $(0.0060)$ & $-500.2 \%$ \\
\hline 61 & 0.0327 & 0.0267 & $(0.0060)$ & $-22.5 \%$ & 131 & 0.0071 & 0.0011 & $(0.0060)$ & $-562.0 \%$ \\
\hline 62 & 0.0316 & 0.0256 & $(0.0060)$ & $-23.4 \%$ & 132 & 0.0070 & 0.0009 & $(0.0060)$ & $-642.0 \%$ \\
\hline 63 & 0.0306 & 0.0246 & $(0.0060)$ & $-24.3 \%$ & 133 & 0.0069 & 0.0008 & $(0.0061)$ & $-751.3 \%$ \\
\hline 64 & 0.0297 & 0.0237 & $(0.0060)$ & $-25.3 \%$ & 134 & 0.0068 & 0.0007 & $(0.0061)$ & $-912.6 \%$ \\
\hline 65 & 0.0288 & 0.0228 & $(0.0060)$ & $-26.3 \%$ & 135 & 0.0067 & 0.0005 & $(0.0061)$ & $-1184.2 \%$ \\
\hline 66 & 0.0279 & 0.0219 & $(0.0060)$ & $-27.3 \%$ & 136 & 0.0066 & 0.0003 & $(0.0062)$ & $-1794.2 \%$ \\
\hline 67 & 0.0271 & 0.0211 & $(0.0060)$ & $-28.4 \%$ & 137 & 0.0065 & 0.0001 & $(0.0064)$ & $-11,232.7 \%$ \\
\hline 68 & 0.0263 & 0.0203 & $(0.0060)$ & $-29.5 \%$ & & & & & \\
\hline 69 & 0.0255 & 0.0195 & $(0.0060)$ & $-30.6 \%$ & & & & & \\
\hline 70 & 0.0248 & 0.0188 & $(0.0060)$ & $-31.7 \%$ & & & & & \\
\hline
\end{tabular}

Table 5. The table shows the Rydberg formula predictions and the relativistic predictions for the first 137 elements. As we can see, the difference increases between the two models. Here we are just looking at the case $n_{1}=1$ and $n_{2}=2$.

\begin{tabular}{ccccccccccc}
\hline $\begin{array}{c}\text { Atomic } \\
\#\end{array}$ & $\begin{array}{c}\text { Rydberg } \\
\text { formula }\end{array}$ & $\begin{array}{c}\text { Relativistic } \\
\text { formula }\end{array}$ & Diff. & $\begin{array}{c}\text { Diff. } \\
\%\end{array}$ & $\begin{array}{c}\text { Atomic } \\
\#\end{array}$ & $\begin{array}{c}\text { Rydberg } \\
\text { formula }\end{array}$ & $\begin{array}{c}\text { Relativistic } \\
\text { formula }\end{array}$ & $\begin{array}{c}\text { Diff. } \\
\%\end{array}$ & $\begin{array}{c}\text { Diff. } \\
\%\end{array}$ \\
\hline 1 & 121.5023 & 121.4962 & -0.0061 & $-0.0050 \%$ & 71 & 0.0241 & 0.0144 & -0.0097 & $-67.6 \%$ \\
2 & 30.3756 & 26.0315 & -4.3440 & $-16.7 \%$ & 72 & 0.0234 & 0.0139 & -0.0096 & $-68.9 \%$ \\
\hline
\end{tabular}




\section{Continued}

\begin{tabular}{|c|c|c|c|c|c|c|c|c|c|}
\hline 3 & 13.5003 & 11.0414 & -2.4589 & $-22.3 \%$ & 73 & 0.0228 & 0.0134 & -0.0094 & $-70.2 \%$ \\
\hline 4 & 7.5939 & 6.0710 & -1.5229 & $-25.1 \%$ & 74 & 0.0222 & 0.0129 & -0.0093 & $-71.6 \%$ \\
\hline 5 & 4.8601 & 3.8329 & -1.0272 & $-26.8 \%$ & 75 & 0.0216 & 0.0125 & -0.0091 & $-73.0 \%$ \\
\hline 6 & 3.3751 & 2.6374 & -0.7377 & $-28.0 \%$ & 76 & 0.0210 & 0.0121 & -0.0090 & $-74.5 \%$ \\
\hline 7 & 2.4796 & 1.9247 & -0.5549 & $-28.8 \%$ & 77 & 0.0205 & 0.0116 & -0.0088 & $-76.0 \%$ \\
\hline 8 & 1.8985 & 1.4659 & -0.4326 & $-29.5 \%$ & 78 & 0.0200 & 0.0112 & -0.0087 & $-77.6 \%$ \\
\hline 9 & 1.5000 & 1.1533 & -0.3467 & $-30.1 \%$ & 79 & 0.0195 & 0.0109 & -0.0086 & $-79.2 \%$ \\
\hline 10 & 1.2150 & 0.9308 & -0.2842 & $-30.5 \%$ & 80 & 0.0190 & 0.0105 & -0.0085 & $-80.9 \%$ \\
\hline 11 & 1.0042 & 0.7668 & -0.2373 & $-31.0 \%$ & 81 & 0.0185 & 0.0101 & -0.0084 & $-82.6 \%$ \\
\hline 12 & 0.8438 & 0.6425 & -0.2013 & $-31.3 \%$ & 82 & 0.0181 & 0.0098 & -0.0083 & $-84.4 \%$ \\
\hline 13 & 0.7189 & 0.5460 & -0.1729 & $-31.7 \%$ & 83 & 0.0176 & 0.0095 & -0.0082 & $-86.2 \%$ \\
\hline 14 & 0.6199 & 0.4696 & -0.1503 & $-32.0 \%$ & 84 & 0.0172 & 0.0092 & -0.0081 & $-88.1 \%$ \\
\hline 15 & 0.5400 & 0.4081 & -0.1319 & $-32.3 \%$ & 85 & 0.0168 & 0.0088 & -0.0080 & $-90.1 \%$ \\
\hline 16 & 0.4746 & 0.3579 & -0.1168 & $-32.6 \%$ & 86 & 0.0164 & 0.0085 & -0.0079 & $-92.2 \%$ \\
\hline 17 & 0.4204 & 0.3163 & -0.1042 & $-32.9 \%$ & 87 & 0.0161 & 0.0083 & -0.0078 & $-94.3 \%$ \\
\hline 18 & 0.3750 & 0.2815 & -0.0935 & $-33.2 \%$ & 88 & 0.0157 & 0.0080 & -0.0077 & $-96.5 \%$ \\
\hline 19 & 0.3366 & 0.2521 & -0.0845 & $-33.5 \%$ & 89 & 0.0153 & 0.0077 & -0.0076 & $-98.8 \%$ \\
\hline 20 & 0.3038 & 0.2270 & -0.0768 & $-33.8 \%$ & 90 & 0.0150 & 0.0075 & -0.0075 & $-101.2 \%$ \\
\hline 21 & 0.2755 & 0.2054 & -0.0701 & $-34.1 \%$ & 91 & 0.0147 & 0.0072 & -0.0075 & $-103.7 \%$ \\
\hline 22 & 0.2510 & 0.1867 & -0.0643 & $-34.5 \%$ & 92 & 0.0144 & 0.0070 & -0.0074 & $-106.3 \%$ \\
\hline 23 & 0.2297 & 0.1704 & -0.0593 & $-34.8 \%$ & 93 & 0.0140 & 0.0067 & -0.0073 & $-109.0 \%$ \\
\hline 24 & 0.2109 & 0.1561 & -0.0548 & $-35.1 \%$ & 94 & 0.0138 & 0.0065 & -0.0073 & $-111.8 \%$ \\
\hline 25 & 0.1944 & 0.1436 & -0.0509 & $-35.4 \%$ & 95 & 0.0135 & 0.0063 & -0.0072 & $-114.7 \%$ \\
\hline 26 & 0.1797 & 0.1324 & -0.0473 & $-35.8 \%$ & 96 & 0.0132 & 0.0061 & -0.0071 & $-117.7 \%$ \\
\hline 27 & 0.1667 & 0.1225 & -0.0442 & $-36.1 \%$ & 97 & 0.0129 & 0.0058 & -0.0071 & $-120.9 \%$ \\
\hline 28 & 0.1550 & 0.1136 & -0.0414 & $-36.5 \%$ & 98 & 0.0127 & 0.0056 & -0.0070 & $-124.2 \%$ \\
\hline 29 & 0.1445 & 0.1056 & -0.0389 & $-36.8 \%$ & 99 & 0.0124 & 0.0054 & -0.0070 & $-127.7 \%$ \\
\hline 30 & 0.1350 & 0.0984 & -0.0366 & $-37.2 \%$ & 100 & 0.0122 & 0.0053 & -0.0069 & $-131.3 \%$ \\
\hline 31 & 0.1264 & 0.0919 & -0.0346 & $-37.6 \%$ & 101 & 0.0119 & 0.0051 & -0.0068 & $-135.1 \%$ \\
\hline 32 & 0.1187 & 0.0860 & -0.0327 & $-38.0 \%$ & 102 & 0.0117 & 0.0049 & -0.0068 & $-139.1 \%$ \\
\hline 33 & 0.1116 & 0.0806 & -0.0310 & $-38.4 \%$ & 103 & 0.0115 & 0.0047 & -0.0067 & $-143.3 \%$ \\
\hline 34 & 0.1051 & 0.0757 & -0.0294 & $-38.8 \%$ & 104 & 0.0112 & 0.0045 & -0.0067 & $-147.7 \%$ \\
\hline 35 & 0.0992 & 0.0712 & -0.0280 & $-39.3 \%$ & 105 & 0.0110 & 0.0044 & -0.0067 & $-152.3 \%$ \\
\hline 36 & 0.0938 & 0.0671 & -0.0267 & $-39.7 \%$ & 106 & 0.0108 & 0.0042 & -0.0066 & $-157.2 \%$ \\
\hline 37 & 0.0888 & 0.0633 & -0.0254 & $-40.2 \%$ & 107 & 0.0106 & 0.0040 & -0.0066 & $-162.4 \%$ \\
\hline 38 & 0.0841 & 0.0598 & -0.0243 & $-40.7 \%$ & 108 & 0.0104 & 0.0039 & -0.0065 & $-167.9 \%$ \\
\hline 39 & 0.0799 & 0.0566 & -0.0233 & $-41.2 \%$ & 109 & 0.0102 & 0.0037 & -0.0065 & $-173.7 \%$ \\
\hline 40 & 0.0759 & 0.0536 & -0.0223 & $-41.7 \%$ & 110 & 0.0100 & 0.0036 & -0.0065 & $-179.8 \%$ \\
\hline 41 & 0.0723 & 0.0508 & -0.0214 & $-42.2 \%$ & 111 & 0.0099 & 0.0034 & -0.0064 & $-186.3 \%$ \\
\hline
\end{tabular}




\section{Continued}

\begin{tabular}{|c|c|c|c|c|c|c|c|c|c|}
\hline 42 & 0.0689 & 0.0483 & -0.0206 & $-42.7 \%$ & 112 & 0.0097 & 0.0033 & -0.0064 & $-193.3 \%$ \\
\hline 43 & 0.0657 & 0.0459 & -0.0199 & $-43.3 \%$ & 113 & 0.0095 & 0.0032 & -0.0064 & $-200.8 \%$ \\
\hline 44 & 0.0628 & 0.0436 & -0.0191 & $-43.9 \%$ & 114 & 0.0093 & 0.0030 & -0.0063 & $-208.8 \%$ \\
\hline 45 & 0.0600 & 0.0415 & -0.0185 & $-44.4 \%$ & 115 & 0.0092 & 0.0029 & -0.0063 & $-217.3 \%$ \\
\hline 46 & 0.0574 & 0.0396 & -0.0178 & $-45.1 \%$ & 116 & 0.0090 & 0.0028 & -0.0063 & $-226.6 \%$ \\
\hline 47 & 0.0550 & 0.0378 & -0.0172 & $-45.7 \%$ & 117 & 0.0089 & 0.0026 & -0.0062 & $-236.6 \%$ \\
\hline 48 & 0.0527 & 0.0360 & -0.0167 & $-46.3 \%$ & 118 & 0.0087 & 0.0025 & -0.0062 & $-247.4 \%$ \\
\hline 49 & 0.0506 & 0.0344 & -0.0162 & $-47.0 \%$ & 119 & 0.0086 & 0.0024 & -0.0062 & $-259.2 \%$ \\
\hline 50 & 0.0486 & 0.0329 & -0.0157 & $-47.7 \%$ & 120 & 0.0084 & 0.0023 & -0.0062 & $-272.1 \%$ \\
\hline 51 & 0.0467 & 0.0315 & -0.0152 & $-48.4 \%$ & 121 & 0.0083 & 0.0021 & -0.0062 & $-286.3 \%$ \\
\hline 52 & 0.0449 & 0.0301 & -0.0148 & $-49.1 \%$ & 122 & 0.0082 & 0.0020 & -0.0061 & $-302.0 \%$ \\
\hline 53 & 0.0433 & 0.0289 & -0.0144 & $-49.8 \%$ & 123 & 0.0080 & 0.0019 & -0.0061 & $-319.5 \%$ \\
\hline 54 & 0.0417 & 0.0277 & -0.0140 & $-50.6 \%$ & 124 & 0.0079 & 0.0018 & -0.0061 & $-339.1 \%$ \\
\hline 55 & 0.0402 & 0.0265 & -0.0136 & $-51.4 \%$ & 125 & 0.0078 & 0.0017 & -0.0061 & $-361.3 \%$ \\
\hline 56 & 0.0387 & 0.0255 & -0.0133 & $-52.2 \%$ & 126 & 0.0077 & 0.0016 & -0.0061 & $-386.6 \%$ \\
\hline 57 & 0.0374 & 0.0244 & -0.0130 & $-53.0 \%$ & 127 & 0.0075 & 0.0015 & -0.0061 & $-415.8 \%$ \\
\hline 58 & 0.0361 & 0.0235 & -0.0126 & $-53.8 \%$ & 128 & 0.0074 & 0.0013 & -0.0061 & $-450.0 \%$ \\
\hline 59 & 0.0349 & 0.0226 & -0.0123 & $-54.7 \%$ & 129 & 0.0073 & 0.0012 & -0.0061 & $-490.7 \%$ \\
\hline 60 & 0.0338 & 0.0217 & -0.0121 & $-55.6 \%$ & 130 & 0.0072 & 0.0011 & -0.0061 & $-540.2 \%$ \\
\hline 61 & 0.0327 & 0.0209 & -0.0118 & $-56.6 \%$ & 131 & 0.0071 & 0.0010 & -0.0061 & $-602.1 \%$ \\
\hline 62 & 0.0316 & 0.0201 & -0.0115 & $-57.5 \%$ & 132 & 0.0070 & 0.0009 & -0.0061 & $-682.3 \%$ \\
\hline 63 & 0.0306 & 0.0193 & -0.0113 & $-58.5 \%$ & 133 & 0.0069 & 0.0008 & -0.0061 & $-791.8 \%$ \\
\hline 64 & 0.0297 & 0.0186 & -0.0111 & $-59.5 \%$ & 134 & 0.0068 & 0.0006 & -0.0061 & $-953.2 \%$ \\
\hline 65 & 0.0288 & 0.0179 & -0.0108 & $-60.6 \%$ & 135 & 0.0067 & 0.0005 & -0.0062 & $-1224.9 \%$ \\
\hline 66 & 0.0279 & 0.0173 & -0.0106 & $-61.7 \%$ & 136 & 0.0066 & 0.0003 & -0.0062 & $-1835.0 \%$ \\
\hline 67 & 0.0271 & 0.0166 & -0.0104 & $-62.8 \%$ & 137 & 0.0065 & 0.0001 & -0.0064 & $-11,273.7 \%$ \\
\hline 68 & 0.0263 & 0.0160 & -0.0102 & $-63.9 \%$ & & & & & \\
\hline 69 & 0.0255 & 0.0155 & -0.0101 & $-65.1 \%$ & & & & & \\
\hline 70 & 0.0248 & 0.0149 & -0.0099 & $-66.3 \%$ & & & & & \\
\hline
\end{tabular}

is consistent with the standard relativistic energy momentum relation, and the Suto formula is based on an alternative theory, with a modified relativistic energy momentum formula. It is too early to say whether or not these relativistic extensions of the Rydberg formula can tell us anything new that is consistent with observations, as it is likely that relativistic quantum mechanical corrections would be needed for that. We encourage others to look further into this, and we hope to do so some time in the future as well.

\section{Acknowledgements}

Thanks to Victoria Terces for helping me edit this manuscript. 


\section{Conflicts of Interest}

The author declares that there is no conflict of interest regarding the publication of this paper.

\section{References}

[1] Haug, G.E. (2020) Journal of Modern Physics, 110, 528-534. https://doi.org/10.4236/jmp.2020.114035

[2] Suto, K. (2020) Journal of Modern Physics, 11, 294-303. https://doi.org/10.4236/jmp.2020.112018

[3] Drake, G.W.F. and Yan, Z.C. (1992) Physical Review A, 460, 2378. https://doi.org/10.1103/PhysRevA.46.2378

[4] Le Bigot, E.O., Jentschura, U.D., Evers, J., Mohr, P.J. and Keitel, C.H. (2005) Journal of Physics B: Atomic, Molecular and Optical Physics, 380, 97. https://doi.org/10.1088/0953-4075/38/2/008

[5] Rydberg, J.R. (1890) Philosophical Magazine, 29, 331-337. https://doi.org/10.1080/14786449008619945

[6] Compton, A.H. (1923) Physical Review, 210, 483. https://doi.org/10.1103/PhysRev.21.483 https://journals.aps.org/pr/abstract/10.1103/PhysRev.21.483

[7] Dirac, P. (1928) Proceedings of the Royal Society of London, 117, 610. https://doi.org/10.1098/rspa.1928.0023

[8] Spavieri, G., Rodriguez, M. and Sanchez, A. (2018) Journal of Physics Communications, 80, 1. https://doi.org/10.1088/2399-6528/aad5fa

[9] Spavieri, G., Gillies, G., Haug, E.G. and Sanchez, A. (2019) Journal of Modern Optics, 66, 2131-2141. https://doi.org/10.1080/09500340.2019.1695005 